\title{
Substitutionsbehandlungen in europäischen Gefängnissen
}

\author{
H. Stöver \\ J. Casselman
}

\section{Maintenance Treatment in European Prisons}

\section{Zusammenfassung}

Ziele: Das Ziel dieser Studie war die Untersuchung der Praxis und Politik der Substitutionsbehandlung in Gefängnissen 18 europäischer Länder. Methodik: In Gefängnissen in 15 europäischen EU Mitgliedsländern (vor dem 1. Mai 2004), sowie in Tschechien, Polen und Slowenien wurden qualitative Interviews mit Vertretern zuständiger Ministerien, Bediensteten der Gefängnisse und Mitarbeitern anderer im Gefängnis aktiven Einrichtungen durchgeführt. Darüber hinaus fanden Interviews in 33 Fokusgruppen mit insgesamt 132 männlichen und 52 weiblichen Gefangenen statt. Ergebnisse: Nachdem die Zugangsbeschränkungen zur Substitutionsbehandlung für nur wenige Zielgruppen (z. B. HIV-positive Opioidkonsumenten) überwiegend abgebaut worden sind, werden Substitutionsbehandlungen nun einem größeren Teil von in Frage kommenden Gefangenen angeboten. Diese Behandlungsform erfüllt allerdings bei weitem nicht die Standards, die an die Substitutionsbehandlung außerhalb der Gefängnisse angelegt werden (v.a. Zugang zur und Kontinuität der Behandlung). In den meisten Ländern wird die in der Gemeinde durchgeführte Substitutionsbehandlung mit Eintreten in das Gefängnis abgebrochen. Es kann eine Behandlungslücke festgestellt werden zwischen Behandlungswunsch vieler Gefangener und Behandlungswirklichkeit. In Europa, z. T. sogar innerhalb einzelner Länder selbst kann eine Heterogenität und Inkonsistenz bezüglich der Richtlinien und Behandlungspraxis festgestellt werden. Die konkrete Vergabepraxis der Substitutionsbehandlung in Gefängnissen variiert von Land zu Land, von Gefängnis zu Gefängnis, innerhalb eines medizinischen Teams, z.T. sogar von Arzt zu Arzt. Obwohl eine psychosoziale Betreuung als ein wertvoller zusätzlicher und notwendiger Teil zur Unterstützung des medizinischen Teils der Behandlung angesehen wurde, wird eine solche Unterstützung nur

\section{Abstract}

Objectives: The objective of this study was to examine practices and policies in place for the provision of substitution treatment in prison in 18 European countries. Methodology: Across the 15 European member states (prior to 1 May 2004) and Czech Republic, Poland and Slovenia, interviews with ministerial representatives, professionals (i.e. service providers and security officials) working in prison, and a total of 33 focus groups with a total of 132 male and 52 female prisoners were conducted. Results: Though constraints of access to substitution treatment for specific target groups only (e.g. HIV-positive opiate users) have largely vanished, substitution treatment is now offered to a broad cross-section of prisoners. The provision of this treatment still lags behind the standards of substitution treatment in the community (regarding access and continuity). In most countries, this form of therapy is most likely to be discontinued when entering prison. A treatment gap persists between prisoners requiring substitution maintenance treatment and those receiving it. Heterogeneous and inconsistent regulations and treatment modalities appear throughout European countries and prisons, sometimes within the same country or region. The concrete provision practice of substitution treatment in prison varies from one country to the other, from one prison to the other, within a medical team, and even from one doctor to the other. Although psychosocial care was seen as a valuable additional and necessary part of the treatment to support the medical part of the substitution treatment in prison, it was found that such support was rarely provided. Nevertheless, compared to previous research, this study illustrates that the scope of substitution treatment has extended considerably across Europe. Across the board, a consensus surrounding the need to continue substitution treatment that had already been started in the community was apparent.

Institutsangaben

Bremer Institut für Drogenforschung (BISORG), Universität Bremen, FB 06 ARCHIDO

Korrespondenzadresse

Dr. Heino Stöver · Bremer Institut für Drogenforschung (BISORG), Universität Bremen, FB 06 ARCHIDO .

Postfach $330440 \cdot 28334$ Bremen·E-mail: heino.stoever@uni-bremen.de

Bibliografie

Suchttherapie 2006; 7: 66 - 71 (c) Georg Thieme Verlag KG Stuttgart · New York

DOI 10.1055/s-2006-926783

ISSN 1439-9903 
selten gewährt. Trotz dieser Einschränkungen zeigt die vorliegende Studie, dass die Verbreitung der Substitutionsbehandlung in europäischen Gefängnissen im Vergleich zu früheren Untersuchungen beträchtlich gestiegen ist. Es konnte ein Konsens darüber festgestellt werden, dass eine außerhalb des Gefängnisses begonnene Substitutionsbehandlung in Haft fortgesetzt werden sollte.

Schliusselwörter

Substitutionsbehandlung · Gefängnis · Methadon · Buprenorphin
Key words

Substitution treatment $\cdot$ prison $\cdot$ methadone $\cdot$ buprenorphine

\section{Einleitung}

Die Substitutionsbehandlung ist in den letzten 20 Jahren zu einer weit verbreiteten und weithin akzeptierten Behandlungsform Opioidabhängiger in Europa avanciert ${ }^{1}$ [1]. Allein in den Jahren 1993-1999 hat sich die Zahl der Therapieplätze verdreifacht. Vom Jahre 2000-2005 hat sich die Zahl von 300000 auf über 550000 fast verdoppelt ${ }^{2}$, d.h. es ergibt sich heute eine „Substitutionsrate“ (in Bezug auf die Anzahl „problematischer Drogengebraucher") von 28-31\%, mit erheblichen Variationsbreiten (von $7 \%$ in Schweden bis hin zu über $58 \%$ in Frankreich [2]. Die Zahl der Substituierten in den 10 „neuen“ EU-Ländern liegt bei etwa 8000 , was eine bisher noch schwache Substitutionsrate von 5-7\% ergibt. Die Gründe dafür sind u.a. in der lange vorherrschenden und beginnenden Auflösung des Abstinenzparadigmas als Kernziel therapeutischer Intervention und einem biologistischen Suchtverständnis [3, 4]. Für die beiden EU-Erwartungsländer Bulgarien und Rumänien lagen keine Informationen vor. In Norwegen wurden im Jahre 20032431 Substituierte aktuell in Behandlung gezählt, in der Schweiz 14458 (2004) [5].

Die Wirksamkeit der Substitutionsbehandlung bei Opioidabhängigen ist in vielen Forschungsarbeiten belegt (siehe www.archido.de, www.indro-online.de). Wissenschaftliche Erkenntnisse und nunmehr langjährige praktische Erfahrungen liegen vor, die zeigen, dass Substitution

- eine sichere Behandlungsform darstellt,

- eine Reduktion des Heroinkonsums und eine Reduktion der Mortalität und Morbidität (z.B. HIV-Transmissionen) bewirkt,

- effektiv darin ist, opioidabhängige Menschen in Behandlung zu halten,

- das Risiko einer HIV-Infektion minimiert,

- sowohl die physische als auch die psychische Gesundheit ebenso wie die Lebensqualität der Patienten steigert,

- zu einer Reduktion der Kriminalität und der Wiederinhaftierung beiträgt,

- kostengünstig ist,

1 Die folgenden Zahlen basieren - soweit nicht anders angegeben - auf den Daten im Jahresbericht 2005 des European Monitoring Centre for Drugs and Drug Addiction, in Lissabon/Portugal (www.emcdda.eu.int).

215 EU-Länder vor dem 1.5.2004. Da für Finnland keine Informationen vorlagen, Substitutionsbeahndlungen jedoch durchgeführt werden und die Zahlen aus der Schweiz nicht mitgezählt werden, liegt die Zahl erheblich über 550000 . und zu positiven Ergebnissen innerhalb verschiedener kultureller Zusammenhänge führen kann (USA, Europa, Asien) [6-8].

Einige Studien zur Bedeutung von Substitutionsbehandlungen im Justizvollzug (v.a. in Australien durchgeführt) haben gezeigt [9], dass Patienten

1. ihren Heroingebrauch, Injektionen und die Gemeinsambenutzung von Spritzen und Nadeln reduzieren,

2. weniger in Drogenhandel im Gefängnis involviert sind,

3. in geringerem Maße unmittelbar nach Haftentlassung versterben,

4. sich in höherem Maße in weiterführende Drogenbehandlungen begeben,

5. die dauerhaft substituiert werden, deutlich weniger drogenbezogene Kriminalität begehen und weniger generell niedrigere Rückfallquoten aufwiesen.

Darüber hinaus profitiert auch das Gefängnis von der Substitutionsbehandlung:

1. besser kontrollierbare Entzugssymptomatik,

2. reduzierter Drogenhandel,

3. erhöhte Produktivität und Arbeitsfähigkeit von Gefangenen.

Was aber passiert genau, wenn Drogenabhängige mit oder ohne Substitutionsbehandlung in totalen Institutionen wie Maßregeloder Strafvollzug untergebracht werden? Trotz der oben skizzierten Erfolge wird die Substitutionsbehandlung nur in geringem Maße in Gefängnissen angeboten bzw. durchgeführt. Die oben genannte Substitutionsrate in Freiheit von 28-31\% wird jedenfalls, bis auf wenige Ausnahmen (z.B. Dänemark und Spanien) weit unterschritten (siehe Tab.1). Selbst in Ländern mit hohen Zahlen von Substitutionsbehandlungen (z.B. Frankreich) geht die Zahl der Substituierten bei Haftantritt dramatisch zurück. Wird die Behandlung durchgeführt, ist sie oftmals Gegenstand von Konflikten zwischen Patient/Innen und Arzt/Ärztin bzw. Krankenpflegepersonal, was Dosis, psychosoziale Begleitung, Ausgabemodi etc. anbetrifft. Eine unabhängige Instanz zur Qualitätssicherung existiert nicht. Dies war der Hintergrund für die von der EU geförderte Studie zur Untersuchung der Substitutionsbehandlung in europäischen Gefängnissen.

\section{Ziele der Studie}

Ziel der Studie war es, die Hürden für die Einführung oder Fortführung der Substitutionsbehandlung in Haft zu analysieren und die 
Tab. 1 Medikamentengestützte Behandlung Opioidabhängiger in Freiheit und in Gefängnissen (18 Länder) ${ }^{1}$

\begin{tabular}{|c|c|c|c|c|c|c|c|}
\hline Land & $\begin{array}{l}\text { Problematischer } \\
\text { Drogenkonsum }\end{array}$ & $\begin{array}{l}\text { Patienten in medika- } \\
\text { menten-gestützter } \\
\text { Behandlung }\end{array}$ & $\begin{array}{l}\text { Substitutions- } \\
\text { rate }^{2}(\%)\end{array}$ & $\begin{array}{l}\text { Zahl der } \\
\text { Gefangenen }\end{array}$ & $\begin{array}{l}\text { Problematischer } \\
\text { Drogenkonsum } \\
\text { im Gefängnis }\end{array}$ & $\begin{array}{l}\text { Zahl der Patienten in Sub- } \\
\text { stitutionsbehandlung }\end{array}$ & $\begin{array}{l}\text { Substitutions- } \\
\text { rate im } \\
\text { Gefängnis }\end{array}$ \\
\hline Belgien & $\begin{array}{l}23200-28400 \\
(2004)\end{array}$ & $1994(2003)$ & $7,0-8,6 \%$ & $\begin{array}{l}9245 \\
(1.3 .2004)\end{array}$ & $4622(50 \%)$ & $224(2004)$ & $5 \%$ \\
\hline Dänemark & $\begin{array}{l}23757-27331 \\
(2004)\end{array}$ & $5528(2003)$ & $20,2-23,2 \%$ & $\begin{array}{l}3908 \\
(25.11 .2003)\end{array}$ & $\begin{array}{l}547^{3} \\
(14 \%)\end{array}$ & 300 & $55 \%$ \\
\hline Deutschland & $\begin{array}{l}152529-189954 \\
(2004)\end{array}$ & 74764 (2003) & $39,3-49,0 \%$ & $\begin{array}{l}81176 \\
(2003)\end{array}$ & 20294 (25\%) & 700 & $3,5 \%$ \\
\hline Finnland & $\begin{array}{l}1100-14000 \\
(2004)\end{array}$ & $\begin{array}{l}240 \text { (170 Buprenorphine und } \\
70 \text { Methadon) }\end{array}$ & $9-13$ & $\begin{array}{l}3719 \\
(15.4 .2004)\end{array}$ & $2975(80 \%)$ & $45(2001)$ & $1,5 \%$ \\
\hline Frankreich & $\begin{array}{l}147900-182600 \\
(2004)\end{array}$ & $106500(2003)$ & $58,3-72,0 \%$ & $\begin{array}{l}55382 \\
(1.4 .2003)\end{array}$ & $18276(33 \%)$ & $2548(2001)$ & $14 \%$ \\
\hline Griechenland & $\begin{array}{l}15853-21652 \\
(2004)\end{array}$ & $2293(2003)$ & $10,6-14,5 \%$ & $\begin{array}{l}8841 \\
(1.12 .2003)\end{array}$ & $27,5 \%^{4}$ & 0 & $0 \%$ \\
\hline Irland & $\begin{array}{l}13405-15819 \\
(2004)\end{array}$ & $8243(2003)$ & $52,1-61,4 \%$ & 3602 & $\begin{array}{l}1080 \\
(30 \% \text { in } 2000)\end{array}$ & 500 & $46 \%$ \\
\hline Italien & $\begin{array}{l}275698-298892 \\
(2004)\end{array}$ & $90738(2003)$ & $30,3-32,9 \%$ & $\begin{array}{l}56761 \\
(1.9 .2003)\end{array}$ & $15442(27 \%)$ & $1860(31.12 .2003)$ & $12 \%$ \\
\hline Luxemburg & $\begin{array}{l}1801-3948 \\
(2004)\end{array}$ & 864 & $26,7-58,6 \%$ & $\begin{array}{l}498 \\
(1.9 .2003)\end{array}$ & $191(43 \%)^{5}$ & 191 & $100 \%$ \\
\hline Niederlande & $\begin{array}{l}29380-32989 \\
(2004)\end{array}$ & 13505 (2003) & $40,9-45,9$ & 16239 & $\begin{array}{l}5358-8119 \\
(33-50 \%)\end{array}$ & keine Daten & keine Daten \\
\hline Österreich & $\begin{array}{l}29397-33535 \\
(2004)\end{array}$ & $6413(2003)$ & $19,2-21,8 \%$ & $\begin{array}{l}8114 \\
(2003)\end{array}$ & $1623(20 \%)$ & $\begin{array}{l}531 \\
(28.2 .2002)\end{array}$ & $33 \%$ \\
\hline Polen & $33-71000(2004)$ & 700 (Nov. 2003) & $1-2,1 \%$ & $\begin{array}{l}80693 \\
(2003)\end{array}$ & $2662(3,3 \%)^{6}$ & 7 (Nov. 2003) & $0,3 \%$ \\
\hline Portugal & $\begin{array}{l}41720-58980 \\
(2004)\end{array}$ & 12508 (2003) & $21,2-29,9 \%$ & $\begin{array}{l}14060 \\
(1.9 .2003)\end{array}$ & $\begin{array}{l}3515-5900 \\
(25-42 \%)\end{array}$ & 607 (2002) & $10-17 \%$ \\
\hline Schottland & $56000(2002)$ & Keine Daten & & $\begin{array}{l}6803 \\
(20.2 .2004)\end{array}$ & $\begin{array}{l}5238^{7} \\
(77 \%)\end{array}$ & 700 & $13,4 \%$ \\
\hline Schweden & $\begin{array}{l}23500-27300 \\
(2004)\end{array}$ & $2100(2003)$ & $7,7-8,9 \%$ & $\begin{array}{l}6755 \\
(1.10 .2003)\end{array}$ & $4053(60 \%)$ & 0 & $0 \%$ \\
\hline Slowenien & 7399 (2004) & $2860(2003)$ & $38,6 \%$ & 5219 (2003) & $\begin{array}{l}703 \\
(13,47 \%)\end{array}$ & 222 & $32 \%$ \\
\hline Spanien & $\begin{array}{l}182498-214152 \\
(2004)\end{array}$ & $88748(2003)$ & $41,0-48,7 \%$ & $\begin{array}{l}57365 \\
(20.2 .2004)\end{array}$ & $26387(46 \%)^{8}$ & $21642(2001)$ & $82 \%$ \\
\hline Tschechien & $\begin{array}{l}9300-13300 \\
(2004)\end{array}$ & $1772(2003)$ & $13,3-19,0 \%$ & $\begin{array}{l}17429 \\
(16.1 .2004)\end{array}$ & $38,5 \%$ & 0 & $0 \%$ \\
\hline
\end{tabular}

1 Basierend auf den Daten der EMCDDA „Drug Treatment Overviews“ (EMCDDA 2005: http://www.emcdda.eu.int); ergänzt durch berichtete Zahlen in den Interviews der Untersuchung bzw. in offiziellen Publikationen

2 Im Original: „Methadone Assisted Treatment Coverage Rate“, Zahl der Substituierten, bezogen auf die Zahl der „Problematischen Drogenkonsumentlnnen“

3 Estimation for all Danish prison, done once a year. The estimation is divided into drug users in general ( 1316 of all inmates $=38 \%$ in 2000 ; rising from $22 \%$ in 1980 to $27 \%$ in 1990) and hard drug users.

${ }^{4}$ Fotiadou et al. (2004).

${ }^{5}$ Comité de Surveillance du Sida Luxembourg, (2003).

${ }^{6}$ According to a study carried out by J. Sierosawski (2003): 3,3\% of prisoners confirmed intravenous drug use.

$777 \%$ of arrestees tested positive for illegal drugs (includes cannabis) in 2002, when tested on the mandatory drug test upon entrance at the prison.

${ }^{8}$ Ministerio del Interior (2001).

Probleme zu benennen, die Gefangene erfahren, wenn sie diese Behandlung aufnehmen wollen. Um diese Ziele zu erreichen, wurde

1. eine Literaturrecherche über Substitutionsbehandlung im Justiz- und Strafvollzug durchgeführt,

2. eine Bestandsaufnahme der Politik und Praxis der Substitutionsbehandlungen in Gefängnissen vorgenommen,

3. eine Übersicht über nationale und regionale Entwicklungen von Pflegestandards erstellt,

4. Probleme bezogen auf die Fortführung der Behandlung von der Gemeinde in das Gefängnis analysiert,

5. ein Austausch organisiert zwischen Ärzten, Krankenpflegepersonal und Fachdiensten,
6. Beispiele „Guter/empfehlenswerter Praxis“ im Bereich der Substitutionsbehandlung identifiziert.

\section{Methodik}

Die Untersuchung wurde über 18 Monate (von Dezember 2002 bis Mai 2004) durchgeführt. Zunächst wurden eine allgemeine Datensammlung und Sichtung vorhandener Dokumente und Abfrage relevanter Datenbanken vorgenommen. Vor diesem Hintergrund wurden Länderbesuche vereinbart, in denen in jeweils zwei Gefängnissen Interviews einerseits mit Ärzten, Krankenpfle- 
gepersonal und allgemeinen Vollzugsbediensteten, andererseits mit Gefangenen durchgeführt wurden. Da es sich um eine explorative Studie handelte, wurde eine qualitative Interviewmethodik gewählt, um den subjektiven Begründungen, Haltungen und Bedeutungen der Befragten mehr Raum zu geben. Zudem handelte es sich um eine sensible Fragestellung, die im Forschungsdesign insofern berücksichtigt wurde, als dass das Thema „Substitutionsbehandlung“ nicht nur von den Ärzten und Krankenpflegern, sondern auch von den Gefangenen diskutiert wurde (in sog. focus groups). Durch Einbezug aller Beteiligten sollte eine ausgewogenere Sichtweise des aktuellen Geschehens im Gefängnis erreicht werden.

Die Untersuchung fand in 33 Gefängnissen in 17 Ländern statt (15 EU-Mitgliedsländer vor dem 1.5.2004, ohne Luxemburg, zusätzlich Tschechien, Polen und Slowenien). Es wurden 184 Gefangene (132 Männer, 52 Frauen) in 33 Fokusgruppen befragt.

\section{Ergebnisse}

Entsprechend der Entwicklung der Substitutionsbehandlung außerhalb des Gefängnisses war diese Behandlungsform in den untersuchten Gefängnissen zunächst für HIV-Positive und/oder an Aids-Erkrankte oder schwangere Gefangene zugänglich. Obwohl diese enge Beschränkung aufgehoben ist, bleibt das Angebot für diese Behandlungsform in Gefängnissen weit hinter den Entwicklungen außerhalb zurück. Es besteht eine „Behandlungslücke“ zwischen den Gefangenen, die eine Substitutionsbehandlung wünschen und denen, die sie bekommen. In den meisten Ländern existiert also eine unzureichende und sehr lückenhafte Versorgung mit dieser Therapieform. Heterogene und inkonsistente Regelungen und Behandlungsbedingungen lassen sich in den meisten untersuchten Gefängnissen feststellen, sogar innerhalb einzelner Länder oder gar Regionen/Städte.

Trotz aller Beschränkungen hat sich die Zahl der Substitutionsbehandlungen in Haft in den letzten Jahren vergrößert: Lediglich Griechenland und Schweden bieten keine Substitutionsbehandlungen in Haft an.

In den meisten untersuchten Ländern führt die Inhaftierung zu einem Abbruch der in der Gemeinde begonnenen Substitutionsbehandlung. Die Gründe dafür sind:

1. eine grundlegende Abstinenzorientierung der Ärzte,

2. einhergehend damit: die Wahrnehmung von Methadon oder eines anderen Substitutionsmittels als psychoaktive Droge, die ungeeignet zur Therapie Drogenabhängiger ist,

3. ein mangelndes Verständnis von Abhängigkeit als eine chronische Krankheit,

4. begrenzte Ressourcen und Expertise.

Auch bei den Gefangenen ließen sich Widerstände und Ablehnungen der Substitutionsbehandlung ausmachen:

1. Ein Mangel an Verständnis über die Zielsetzung von Substitutionsbehandlung: Der Gefängnisaufenthalt wurde oftmals als (einzige) drogenfreie Zeit betrachtet - in diesem Kontext wurde auch Methadon als „Droge” wahrgenommen, weil sie oftmals auch auf dem Schwarzmarkt erworben wurde, und eher um ihrer psychoaktiven denn therapeutischen Wirkung konsumiert wurde.

2. Gefangene wollen (über die Teilnahme an einem Methadonprogramm) nicht als „drogenabhängig“ erkannt werden, weil sie Nachteile für den Vollzug der eigenen Strafe befürchten.

Unter den meisten Befragten bestand jedoch ein Konsens darüber, dass in der Gemeinde begonnene Substitutionsbehandlungen in Haft fortgeführt werden sollten. In der Praxis war dies neben den o.g. (Ablehnungs-)Gründen häufig abhängig von der bisherigen Behandlungsdauer in der Gemeinde, den in Haft zur Verfügung stehenden Plätzen und der voraussichtlichen Zeit der Inhaftierung. Ein Beginn einer Substitutionsbehandlung in Haft wurde sehr viel problematischer gesehen und Probleme der Überleitung waren in einigen Ländern bedeutsam (z.B. nicht genügend freie Plätze in Substitutionsprogrammen). In einigen Ländern (z.B. Belgien) ist die Substitutionsbehandlung formell begrenzt auf eine Zeit zwischen 6 und 12 Monaten, in einigen anderen Ländern bestand diese Begrenzung informell, ohne in Richtlinien festgeschrieben zu sein.

In einigen Ländern besteht keine zeitliche Limitierung: Substitutionsbehandlungen werden „Kurzstraflern“ ebenso angeboten wie „Lebenslänglichen“: In Österreich und Spanien bspw. ist dies die Standardpraxis.

Obwohl psychosoziale Begleitung als wichtiger und integraler Bestandteil der Behandlung und als sinnvolle Ergänzung zum medizinischen Teil der Therapie betrachtet wird, wird sie in systematischer und koordinierter Form nur in wenigen untersuchten Gefängnissen angeboten.

Eine große Heterogenität besteht in Europa und auch innerhalb eines Landes oder gar Region bezüglich der Entzugsmodalitäten: Reduktionsschemata scheinen von Gefängnis zu Gefängnis und selbst von Arzt zu Arzt zu variieren: von 7 Tagen bis zu 4 Wochen.

Der Gebrauch von Benzodiazepinen und Mehrfachabhängigkeit ist ebenso wie in der Gemeinde als auch im Gefängnis ein Problem für die Substitutionsbehandlung, Entzug und Schwere der Abhängigkeit insgesamt geworden.

Die Studie hat ergeben, dass die durchschnittliche Substitutionsdosis in Haft variierte (zwischen 30 und 70 mg Methadon). Im Gegensatz zur Praxis in der Gemeinde glaubten viele befragte Ärzte und Krankenpfleger, dass Niedrigdosen ausreichend für die Unterdrückung des „craving“ sind. Als Gründe wurden benannt, dass eine $100 \%$ ige Einnahme des Substituts garantiert werden konnte und dass der Umfang zusätzlich eingenommener Drogen im Vergleich zu „draußen“ reduziert werden kann.

Die Information der Gefangenen (z.T. auch der Bediensteten) über Sinn der Substitutionsbehandlung, Wirkung einzelner Substanzen und grundsätzliche Substitutionspolitik war in vielen Gefängnissen mangelhaft. Häufig verstanden Gefangenen weder die Zielsetzung des Substitutionsprogramms, den Sinn eingesetzter Substanzen, Behandlungsschemata noch die Einschluss- und Ausschlusskriterien. Dies stellt ein grundsätzliches Problem dar in Bezug auf die „informierte Zustimmung” des Patienten zur Behandlung. Oftmals wurde von Ärzten und Krankenpflegepersonal die 
Information über die Substitutionsbehandlung als gegeben vorausgesetzt.

Anonymität und Vertraulichkeit ist im Gefängniszusammenhang grundsätzlich schwer einzuhalten; trotzdem wurden in vielen Anstalten Versuche unternommen, die Tatsache der Substitutionsbehandlung eines Gefangenen vertraulich zu behandeln: Entweder wurden alle Substitutionspatienten in einem Flügel untergebracht, oder die konkrete tägliche Abgabe erfolgte diskret zusammen mit anderen Medikamenten. Gefangene berichteten auch über Ausnahmen, in denen ihre Teilnahme am Substitutionsprogramm öffentlich für jedermann (Mitgefangene und Bedienstete) einsehbar war (z.B. durch Kennzeichnung an der Zellentür).

In den meisten untersuchten Ländern gab es keine besondere Ausbildung für Ärzte und Krankenpflegepersonal im Gefängnis. Da sich viele neue medizinisch-pflegerische Herausforderungen angesichts der gesundheitlichen Belastungen vieler Gefangener stellen, wurden spezifische Fortbildungsveranstaltungen im Allgemeinen begrüßt. Lediglich in einigen Ländern benötigen Ärzte zur Verschreibung von Opiaten eine besondere (Zusatz-)Ausbildung; dies wurde als ein wichtiger Schritt zur Steigerung der Behandlungsqualität betrachtet.

\section{Ergebnisse für Deutschland: heterogene Substitutionspraxis}

Trotz bundeseinheitlicher rechtlicher Rahmenbedingungen ist die Substitution in den Gefängnissen Deutschlands in der Praxis sowohl hinsichtlich Ziel und Zweck als auch hinsichtlich Häufigkeit sehr unterschiedlich gestaltet. Selbst länderspezifische Verwaltungsvorschriften werden in den Gefängnissen der betreffenden Länder uneinheitlich umgesetzt. So begegnet man in Deutschland einer sehr heterogenen Substitutionspraxis, die von Bundesland zu Bundesland, von Gefängnis zu Gefängnis und z. T. von Arzt zu Arzt unterschiedlich gehandhabt wird. Von einer flächendeckenden Versorgung kann nur in wenigen Bundesländern gesprochen werden, eine zeitliche Befristung (auf 3-6 Monate) ist darüber hinaus weit verbreitet.

Der Umfang der medizinischen Versorgung der Inhaftierten ist im Strafvollzugsgesetz im Kapitel Gesundheitsfürsorge ( $\S 56-66$ ) festgelegt. Immer wieder (zum Teil wörtlich) verweist der Gesetzgeber hier auf die Vorgaben der Gesetzlichen Krankenversicherung (GKV). Tenor des Gesetzes ist in diesen Paragraphen eindeutig eine Angleichung der gefängnismedizinischen Versorgung an die Versorgung der in der GKV Versicherten. Dennoch gelten für die Substitution in Gefängnissen die BUB-Richtlinien der GKV allenfalls über einen Umweg. So ist in einigen Bundesländern die Methadonsubstitution über Erlasse dergestalt geregelt, dass ausdrücklich auf die Vorgaben der BUB-Richtlinien verwiesen wird.

Dennoch kommt es immer zu Schwierigkeiten beim Wechsel von substituierten Patienten aus dem System der GKV in das Gefängnis, aber auch umgekehrt, beim Wechsel nach Haftentlassung vom Gefängnis in die GKV [9].

\section{Diskussion}

Um eine patientenorientierte Behandlung mit höherer Qualität zu erreichen, sollte eine
1. wesentliche Ausdehnung von Substitutionsbehandlungen in den meisten Ländern erfolgen,

2. erhebliche Qualitätsverbesserung der Behandlung eingeleitet warden,

3. Verbesserung der Kommunikation und Kooperation zwischen Gesundheitsdiensten des Gefängnisses und der Gemeinde/Stadt/Region angestrebt werden.

Die Studie zeigte außerdem, dass das Ziel der Erreichung einer Drogenfreiheit für alle Gefangenen das Erreichen anderer wesentlicher und existenziell wichtiger Ziele, wie Vermeidung von HIV-/ Hepatitis-Infektionen, Überdosierung nach Haftentlassung, gefährdet. Im Rahmen einer Prioritätensetzung muss die Gefahr irreversible Schädigungen an oberster Stelle gesetzt werden.

Niedrig- und hochschwellige Programme sollten erwogen werden, die einerseits „harm reduction“-Ziele verfolgen und andererseits mit zusätzlichen Mitteln für psychosoziale Begleitung weiterführende Therapieziele verfolgen.

Die besonderen Behandlungsbedürfnisse von drogenabhängigen Frauen sollten angesichts der Komplexität und Schwere ihrer Abhängigkeit und weiteren sozialen-gesundheitlichen Belastungen stärker berücksichtigt werden.

Die Expertise und Kompetenz von betroffenen Gefangenen sollte in Zieldiskussion, Gestaltung der Therapie und konkrete Durchführung einbezogen werden: Ausgehend von ihren Bedürfnissen und Erfahrungen in früheren Substitutionsbehandlungen (u.a. in Haft) kann die Qualität und die Wirksamkeit der Behandlungen erhöht werden. Gefangene mit Substitutionserfahrungen sind willens und in der Lage substanziell wertvolle Beiträge zur Verbesserung der Behandlung zu machen.

In vielen Ländern ist die Substitutionsbehandlung in Haft nicht oder nicht ausreichend dokumentiert oder gar überprüft; es existieren oft nur grobe Schätzungen über die Zahl der substituierten Patienten und die Behandlungsmodalitäten. In fast jedem besuchten Land gab es keine wissenschaftlichen Überprüfungen der Behandlungswünsche und -bedürfnisse der Patienten und der Sichtweisen der Behandlungsanbieter.

Zur Qualitätsverbesserung der Behandlung zählt auch eine Intensivierung des Austausches, auf der Basis einer Patientenzustimmung, zwischen behandelndem Arzt/Krankenpflege und entsprechenden Anbietern von Substitutionsbehandlungen am Heimatort der Gefangenen, um Probleme der Fortführung, Übergänge, Anpassungen besser oder überhaupt regeln zu können.

Einige Beispiele „guter/empfehlenswerter Praxis“ wurden identifiziert:

1. Leitlinien eines klinischen Managements der Substitutionsbehandlung,

2. Kommunikations- und Kooperationsstrukturen zwischen allen beteiligten Fachdiensten und Personen im Gefängnis (regelmäßige Treffen und Fallbesprechungen zwischen Ärzten, Krankenpflegern, Sozialarbeitern, Psychologen),

3. Netzwerkarbeit mit den Einrichtungen der Drogen- und AidsHilfe in der Gemeinde. 
Auf der Basis dieser Forschungsergebnisse wurden schließlich Empfehlungen für eine Qualitätsverbesserung der Substitutionsbehandlung ausgesprochen.

\section{Literatur}

${ }^{1}$ Council of Europe. Development and Improvement of Substitution Programmes. Proceedings. Seminar organised by the Co-operation Group to Combat Drug Abuse and Illicit Trafficking in drugs (Pompidou Group). Strasbourg, France, 8. -9.10.2001

2 Gerlach R, Stöver H. Vom Tabu zur Normalität. 20 Jahre Substitutionsbehandlung in Deutschland. Zwischenbilanz und Aufgaben für die Zukunft. Freiburg: Lambertus, 2005

${ }^{3}$ Subata E. Difficulties encountered in post-Soviet countries in accepting the principle of substitution treatment. Council of Europe, a.a.O 2000: 83-106

${ }^{4}$ Habrat B. Difficulties encountered in Central and Eastern European countries in acepoting methadone substitution. Council of Europe, a. a.0 2001: $107-118$
${ }^{5}$ Bundesamt für Gesundheit. Ergebnisse der Nationalen Substitutionsstatistik (Methadon) (http://www.nasuko.ch/nms/db/statistik_overview.cfm?sel_year $=2004 \&$ CFID $=11747823 \&$ CFTOKEN $=56611354)$ am 30.6.2005

${ }^{6}$ Verster A. Substitution Treatment for Opiate Dependence in Europe. Presentation Montego Bay, 2001 (http://www.cicad.oas.org/Reduccion_Demanda/ENG/DRresources/experts/ExpertsIV/Pres-Verstereng.ppt)

${ }^{7}$ Küfner H, Rösner S. Ergebnisse von 40 Jahren weltweiter Substitutionsforschung. In: Gerlach, R.; Stöver, H (Hrsg). Vom Tabu zur Normalität. 20 Jahre Substitutionsbehandlung in Deutschland. Zwischenbilanz und Aufgaben für die Zukunft. Freiburg: Lambertus, 2005

8 WHO/UNODC/UNAIDS. Substitution maintenance therapy in the management of opioid dependence and HIV/AIDS prevention. Joint Position paper 2004

${ }^{9}$ Keppler K, Knorr B, Stöver H. Zum Stand der Substitutionsbehandlung im deutschen Justizvollzug. Zeitschrift für Strafvollzug und Straffälligenhilfe 2004; 53 (H 4): $202-217$ 